\title{
Breath Analysis in Pulmonary Arterial Hypertension
}

\author{
Frank S. Cikach Jr, BS; Adriano R. Tonelli, MD; Jarrod Barnes, PhD; Kelly Paschke, BA; \\ Jennie Newman, LPN; David Grove, PhD; Luma Dababneh, MD; Sihe Wang, MD; \\ and Raed A. Dweik, MD, FCCP
}

\begin{abstract}
Background: Pulmonary arterial hypertension (PAH) is a progressive and devastating condition characterized by vascular cell proliferation and is associated with several metabolic derangements. We hypothesized that metabolic derangements in PAH can be detected by measuring metabolic by-products in exhaled breath.

Methods: We collected breath and blood samples from patients with PAH at the time of right-sided heart catheterization $(n=31)$ and from healthy control subjects $(n=34)$. Breath was analyzed by selected ion flow tube-mass spectrometry in predetermined training and validation cohorts.

Results: Patients with PAH were $51.5 \pm 14$ years old, and 27 were women $(85 \%)$. Control subjects were $38 \pm 13$ years old, and 22 were women $(65 \%)$. Discriminant analysis in the training set identified three ion peaks $\left(\mathrm{H}_{3} \mathrm{O}+29+, \mathrm{NO}+56+\right.$, and $\left.\mathrm{O}_{2}+98+\right)$ and the variable age that correctly classified $88.9 \%$ of the individuals. In an independent validation cohort, $82.8 \%$ of the individuals were classified correctly. The concentrations of the volatile organic compounds 2-propanol, acetaldehyde, ammonia, ethanol, pentane, 1-decene, 1-octene, and 2-nonene were different in patients with PAH compared with control subjects. Exhaled ammonia was higher in patients with PAH (median [interquartile range]: 94.7 parts per billion (ppb) [70-129 ppb] vs $60.9 \mathrm{ppb}[46-77 \mathrm{ppb}], P<.001$ ) and was associated with right atrial pressure $(\rho=0.57, P<.001)$, mean pulmonary artery pressure $(\rho=0.43, P=.015)$, cardiac index by thermodilution $(\rho=-0.39, P=.03)$, pulmonary vascular resistance $(\rho=0.40, P=.04)$, mixed venous oxygen $(\rho=-0.59, P<.001)$, and right ventricular dilation $(\rho=0.42, P=.03)$.
\end{abstract}

Conclusions: Breathprint is different between patients with PAH and healthy control subjects. Several specific compounds, including ammonia, were elevated in the breath of patients with PAH. Exhaled ammonia levels correlated with severity of disease.

CHEST 2014; 145(3):551-558

Abbreviations: $\mathrm{PAH}=$ pulmonary arterial hypertension; RV = right ventricular; SIFT-MS = selected ion flow tube-mass spectrometry; $\mathrm{SvO}_{2}=$ mixed venous oxygen saturation; $\mathrm{VOC}=$ volatile organic compound

$\mathbf{P}$ ulmonary arterial hypertension (PAH) is a progressive and devastating condition characterized by vascular smooth muscle and endothelial cell proliferation leading to vascular narrowing that results in elevated pulmonary artery pressure, right ventricle failure, and premature death. ${ }^{1,2}$ Despite recent advances in the understanding of the disease, most currently used biomarkers are invasive and are based mainly on the hemodynamic consequences of the disease, rather than the underlying pathobiology of cell proliferation. Thus, there is a pressing need to identify consistent and noninvasive biomarkers based on the pathobiology of the disease that can be used in evaluation and management. ${ }^{3}$
Regardless of significant progress in the management of the disease, there is still an average delay of more than 2 years from the time of initiation of symptoms to the confirmation of the diagnosis by rightsided heart catheterization. , $^{4,5}$ Several indicators of $\mathrm{PAH}$ disease severity and prognosis are available and derive from the clinical evaluation, blood work, 6-min walk test, echocardiography and right-sided heart catheterization. ${ }^{6-8}$ Breath analysis is recognized increasingly as a noninvasive method for medical diagnostics based on the measurement of the hundreds of volatile organic compounds (VOCs) present in the breath. ${ }^{9}$ Breath can be analyzed by identifying specific compounds/mass scanning peaks or by employing pattern 
recognition of breath compounds. This has paved the way for commercially available breath-testing devices that measure specific compounds (eg, ethanol, ${ }^{14} \mathrm{C}$-urea, and nitric oxide) that are useful in the screening and monitoring of diseases such as alcohol intoxication, Helicobacter pylori infection, and asthma. ${ }^{10,11}$ Limited information is available on the use of exhaled breath analysis in patients with PAH. Currently, studies of exhaled breath in PAH have focused primarily on exhaled nitric oxide, a molecule that is lower in patients with PAH than in healthy control subjects. ${ }^{12-19}$ However, a study of the VOCs in the breath of patients with PAH has not been reported previously. Furthermore, investigations studying biomarkers such as amino-terminal pro-brain natriuretic peptide and endothelin-1 were performed only on exhaled breath condensate. ${ }^{12,20,21}$ To date, no studies have investigated VOCs in the exhaled breath of patients with $\mathrm{PAH}$, using selected ion flow tube-mass spectrometry (SIFT-MS). Although the use of SIFT-MS for breath analysis in different disease states has been reported previously, to our knowledge, this is the first report of its use in patients with pulmonary hypertension.

SIFT-MS uses precursor ions that react with incoming gas compounds to generate product ions, which are then quantified. This method is fast and allows the collection of online samples of volatile compounds in the exhaled breath for real-time feedback. For several VOCs, the accuracy of the instrument can be down to the parts-per-trillion level. SIFT-MS has the advantage of using three precursor ions, rather than one, to generate a more robust mass spectrum. This process also eliminates the need for special sample preparation.22-24

Using this technology, we conducted a prospective, single-center cohort study to assess whether a characteristic pattern of exhaled compounds is present in

Manuscript received June 12, 2013; revision accepted September 9, 2013.

Affiliations: From the Pathobiology Department (Mr Cikach; Drs Barnes, Grove, Dababneh, and Dweik; and Ms Paschke), Lerner Research Institute; the Department of Pulmonary, Allergy and Critical Care Medicine (Drs Tonelli and Dweik and Ms Newman), Respiratory Institute; and the Department of Clinical Pathology (Dr Wang), Cleveland Clinic, Cleveland, $\mathrm{OH}$

Part of this article has been presented in abstract form at the American Thoracic Society Conference, May 22, 2013, Philadelphia, PA.

Funding/Support: This publication was made possible by the Third Frontier Program of the Ohio Department of Development [Grant BRCP 08-049]. Dr Tonelli is supported by Clinical and Translational Science Awards KL2 [Grant RR024990] from the National Center for Research Resources, a component of the NIH, and by the NIH Roadmap for Medical Research. Dr Dweik is supported by the National Institutes of Health [Grants HL081064, HL107147, and RR026231].

Correspondence to: Raed A. Dweik, MD, FCCP, 9500 Euclid Ave A-90, Cleveland, OH 44195; e-mail: dweikr@ccf.org

(C) 2014 American College of Chest Physicians. Reproduction of this article is prohibited without written permission from the American College of Chest Physicians. See online for more details. DOI: 10.1378/chest.13-1363 patients with $\mathrm{PAH}$, and to correlate VOCs and mass scanning peaks with conventional clinical parameters.

\section{Materials AND Methods}

\section{Study Population}

The study protocol was approved by the Cleveland Clinic institutional review board (protocol number 06-245). Informed consent was obtained from all subjects before enrollment. We enrolled patients who underwent right-sided heart catheterization for the diagnosis or follow-up of PAH between June 2011 and April 2012 (training cohort) and between May 2012 and October 2012 (validation cohort). During the same time period, we recruited healthy control subjects. Control subjects had no known diseases and were receiving no medications

We identified 31 patients with PAH and 34 healthy control subjects. Data comparison from 17 patients with PAH and 19 control subjects served as the training cohort to establish a unique metabolic profile. After these initial results were obtained, we recruited a separate cohort of 14 subjects with PAH and 15 control subjects to validate our initial results. We recorded demographic data, as well as data on 6-min walk test, N-terminal prohormone of brain natriuretic peptide, echocardiography, and right-sided heart catheterization results, on all patients with $\mathrm{PAH}$.

\section{Exhaled Breath Collection}

Breath collection was obtained in the fasting state following a bottled-water mouth rinse to minimize and standardize the contribution of the aerodigestive tract. Breath samples from patients with PAH were acquired immediately after right-sided heart catheterization. Initially, patients were instructed to perform a tidal volume exhalation to clear residual air from the anatomic dead space. Patients were then instructed to breathe in deeply through a clean mouthpiece and filter, followed by a tidal volume exhalation through a sterile mouthpiece while attempting to maintain an exhaled pressure of 15 millibars. Exhaled breath was collected in a Mylar balloon bag and incubated at $37^{\circ} \mathrm{C}$ prior to analysis. Corrections for ambient VOCs were not necessary because of the charcoal scrubber used on the exhaled-breath collection device.

\section{Breath Analysis}

All exhaled breath analyses were performed by SIFT-MS (Syft Technologies). Quantitative assessment of 21 prespecified VOCs and mass scanning of ion products for $\mathrm{H}_{3} \mathrm{O}+, \mathrm{O}_{2}+$, and $\mathrm{NO}+$ from 14 to 200 atomic mass units were performed. Mass scanning peaks were assessed visually for patients with $\mathrm{PAH}$ and control subjects. The selected ion-monitoring mode focused on specific VOCs for a more accurate concentration measurement. Most of the 21 VOCs were selected based on a previously described association in the context of disease. ${ }^{25}$ Some of these 21 VOCs (such as 2-propanol) had unknown endogenous sources. The calculation of VOC ammonia was performed using mass scanning peaks $\mathrm{H}_{3} \mathrm{O}+18+$ and $\mathrm{O}_{2}+17+$.

\section{Ammonia Determination in Plasma}

In 17 patients with PAH and 20 healthy control subjects, we determined ammonia in plasma from venous blood samples collected in an ethylenediaminetetraacetic acid specimen tube. Blood was obtained in a fasting state from an internal jugular central venous line at the time of catheterization (patients with $\mathrm{PAH}$ ) or from peripheral venous puncture (control subjects). Blood was separated within 15 min of collection, and plasma was stored at 
$-80^{\circ} \mathrm{C}$. Ammonia levels in ethylenediaminetetraacetic acid plasma were measured by a US Food and Drug Administration-approved enzymatic method with glutamate dehydrogenase on an Integra 800 analyzer (Roche Diagnostics). The assay had an analytical measurable range of 10 to $700 \mu \mathrm{mol} / \mathrm{L}(17-1192 \mu \mathrm{g} / \mathrm{dL})$ with an analytical precision of $3.6 \%$ or less.

\section{Statistical Analysis}

VOCs were assessed visually for normality and screened for outliers. Continuous variables were compared using the MannWhitney $U$ (Wilcoxon) test. The Spearman rank correlation test was used to compare exhaled breath findings with clinical and hemodynamic characteristics in the PAH group. We used linear regression to adjust the studied variables for age. Group classification (PAH vs control subjects) was performed using canonical discriminant analysis using forward stepwise variable selection of the mass scanning peaks. Assumptions of the discriminant analysis were tested. Canonical correlation represents the multiple correlations between the predictors and the discriminant function and provides an index of overall model fit. ${ }^{26} \mathrm{~A}$ canonical score is a composite product derived from weighing the scores of the variables selected by the discriminant analysis. We used two parsimonious models; one included age in addition to three ion peaks and the other, three VOCs. Three ion peaks $\left(\mathrm{H}_{3} \mathrm{O}+29+, \mathrm{NO}+56+\right.$, and $\left.\mathrm{O}_{2}+98+\right)$, together with the variable age, were used to classify subjects in the training set.

Each mass entered into the model was assessed visually to exclude confounding signals caused by isotope peaks or water clustering of VOCs. These peaks were removed from the analysis because they were generated by secondary chemical reactions rather than by single collision reactions of the compounds. To reduce the possibility of type 1 error, we validated our initial model (training model) using a second cohort (validation model). All analyses were performed using JMP Pro, version 9.0 (SAS Institute) and SPSS, version 17 (IBM Corp).

\section{RESULTS}

\section{Patient Characteristics}

Patients with PAH were $51.5 \pm 14$ years old, and 27 were women $(87 \%)$. The characteristics of the PAH group are summarized in Table 1. Control subjects were $38 \pm 13$ years old, and 22 were women $(65 \%)$.

\section{Categorization of Disease Groups Using Unique Metabolic Breathprint}

The discriminate function revealed a significant association between the groups and all predictors, accounting for $63.3 \%$ of the between-group variability. The model had five misclassifications (three patients with $\mathrm{PAH}$ and two control subjects) with a canonical correlation of 0.74 (Wilks' $\lambda, 0.46 ; P<.001$ ). The crossvalidated classification showed that, overall, $86.1 \%$ of the individuals were classified correctly.

The discriminant analysis model was then tested successfully in an independent validation cohort (14 patients with PAH and 15 control subjects), with six misclassifications (three patients with PAH and three control subjects) with a canonical correlation 0.71 (Wilks' $\lambda, 0.50 ; P<.005$ ). The cross-validated

\section{Table 1-Characteristics of Patients With PAH}

\begin{tabular}{|c|c|}
\hline Characteristics & Value \\
\hline No. & 31 \\
\hline Age, mean (SD), y & $51.5(14)$ \\
\hline Female, (\%) & $27(87)$ \\
\hline \multicolumn{2}{|l|}{ Type of PAH, No. } \\
\hline Idiopathic/heritable & 18 \\
\hline CTD associated & 10 \\
\hline CHD associated & 1 \\
\hline $\mathrm{PoPH}$ & 2 \\
\hline \multicolumn{2}{|l|}{ Race, No. $(\%)$} \\
\hline White & $25(81)$ \\
\hline Black & $5(16)$ \\
\hline Not provided & $1(3)$ \\
\hline \multicolumn{2}{|l|}{ NYHA functional class, No. (\%) } \\
\hline I & $5(16)$ \\
\hline II & $12(39)$ \\
\hline III & $12(39)$ \\
\hline IV & $1(3)$ \\
\hline Patients on oxygen, No. (\%) & $6(19)$ \\
\hline Oxygen flow, mean (SD), L/min & $3(1)$ \\
\hline \multicolumn{2}{|l|}{ 6-min walk test } \\
\hline Distance walked, mean (SD), m & $374(124)$ \\
\hline Distance walked, mean (SD), \% predicted & $68(17)$ \\
\hline \multicolumn{2}{|l|}{ Echocardiogram } \\
\hline RVSP, mean (SD), mm Hg & $73(31)$ \\
\hline \multicolumn{2}{|l|}{ RV function, No. (\%) } \\
\hline Normal & $11(35)$ \\
\hline Mild & $7(23)$ \\
\hline Moderate & $7(23)$ \\
\hline Severe & $6(19)$ \\
\hline \multicolumn{2}{|l|}{ RV dilation, No. (\%) } \\
\hline Normal & $11(35)$ \\
\hline Mild & $8(26)$ \\
\hline Moderate & $6(19)$ \\
\hline Severe & $6(19)$ \\
\hline \multicolumn{2}{|l|}{ Right-sided heart catheterization } \\
\hline RA pressure, $\mathrm{mm} \mathrm{Hg}$ & $7 \pm 5$ \\
\hline PA mean pressure, $\mathrm{mm} \mathrm{Hg}$ & $45 \pm 13$ \\
\hline PAOP, mm Hg & $12 \pm 4$ \\
\hline Cardiac index, $\mathrm{L} / \mathrm{min} / \mathrm{m}^{2}$ & $3 \pm 1$ \\
\hline PVR, Wood units, (SD) & $6.6(3.8)$ \\
\hline $\mathrm{SvO}_{2}, \%$ & $67 \pm 8$ \\
\hline
\end{tabular}

$\overline{\mathrm{CHD}}=$ congenital heart disease $\mathrm{CTD}=$ connective tissue disease, $\mathrm{NYHA}=$ New York Heart Association; PA = pulmonary artery; PAH $=$ pulmonary arterial hypertension; $\mathrm{PAOP}=$ pulmonary artery occlusion pressure; $\mathrm{PoPH}=$ portopulmonary hypertension; $\mathrm{PVR}=$ pulmonary vascular resistance; $\mathrm{RA}=$ right atrial; $\mathrm{RV}=$ right ventricular; $\mathrm{RVSP}=$ right ventricular systolic pressure; $\mathrm{SvO}_{2}=$ mixed venous oxygen saturation.

classification showed that, overall, $79.31 \%$ of the individuals were classified correctly. There were no obvious clinical or other factors to distinguish the misclassified subjects from the others. Figure 1 shows the results of the discriminant analysis for all subjects. In preparation for this study, we also compared the breathprint of patients with $\mathrm{PAH}$ with that of individuals with other lung disease (asthma) and systemic (liver) disease, and found that the PAH breath signature by SIFT-MS was unique (e-Fig 1). 
Correlation Between Mass Scanning Peaks and Clinical Factors

Pulmonary artery occlusion pressure was associated with $\mathrm{H}_{3} \mathrm{O}+29+(\rho=0.54, P=.002), \mathrm{H}_{3} \mathrm{O}+43+$ $(\rho=0.58, P<.001)$, and $\mathrm{O}_{2}+28+(\rho=0.39, P=.03)$. Mass scanning peaks corresponding to ammonia were associated with right atrial pressure, mean pulmonary artery pressure, pulmonary vascular resistance, cardiac index, and mixed venous oxygen saturation $\left(\mathrm{SvO}_{2}\right)$ (Table 2).

\section{VOC Changes in PAH}

Of the 21 prespecified VOCs, the concentrations of 2-nonene, 2-propanol, acetaldehyde, ammonia, ethanol, and pentane were elevated in patients with PAH compared with control subjects (Table 3). There were no significant differences between groups in the exhaled concentrations of acetone, acrylonitrile, benzene, carbon disulfide, dimethyl sulfide, isoprene, 1-nonene, 1-heptene, 3-methylhexane, ethane, hydrogen sulfide, triethyl amine, or trimethyl amine. Sev-

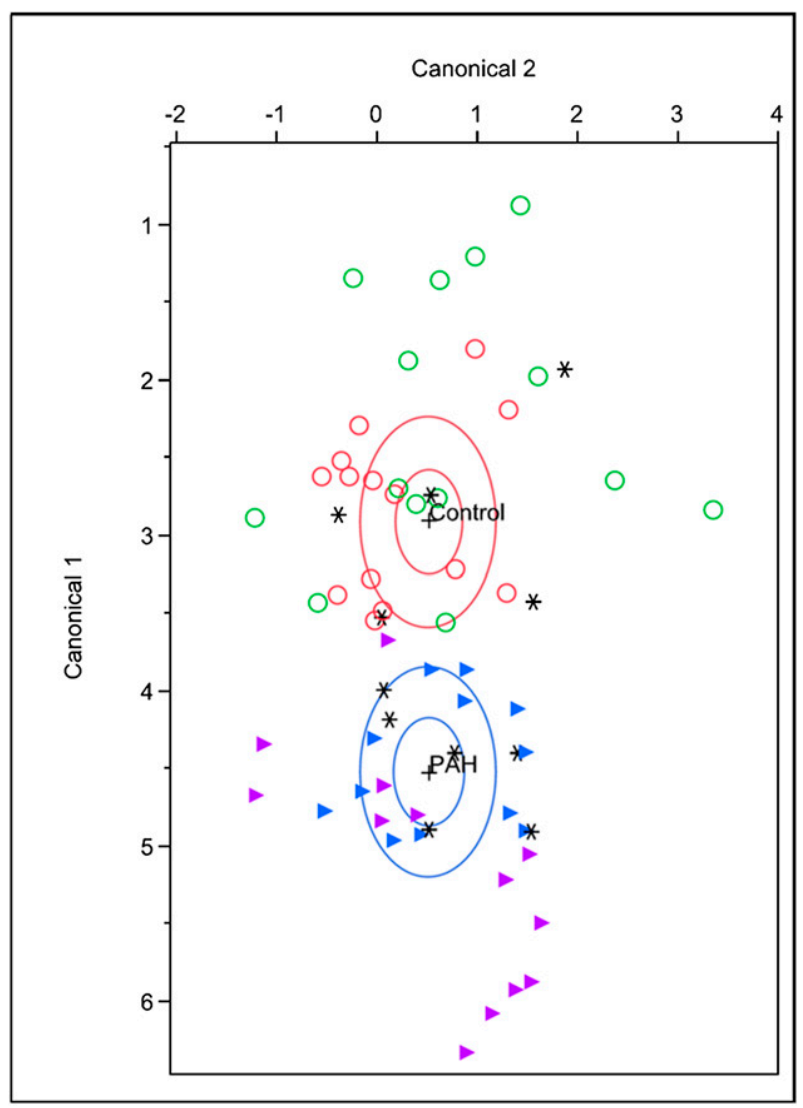

Figure 1. Canonical discriminant analysis was performed in a training cohort of 17 patients with PAH (purple $\triangle$ ) and in 19 control subjects (green O). This PAH "breathprint" was then used to classify an independent validation cohort of 14 patients with PAH (blue $\triangle$ ) and 15 control subjects (red $\bigcirc$ ). * Misclassified individuals. $\mathrm{PAH}=$ pulmonary arterial hypertension. eral VOCs, including 1-decene and 1-octene, were decreased in patients with PAH compared with control subjects. Figure 2 shows selected areas of the average mass spectra for the PAH and control groups.

Using a similar approach, a discriminant analysis using VOCs was conducted to predict whether subjects were in the PAH or the control group. In the training cohort, we identified three VOCs (2-propanol, 1-octene, and ammonia). The number of misclassifications was six $(16.7 \%)$ (canonical correlation, 0.67; Wilks' $\lambda, 0.55 ; P<.001$ ). Using the same VOCs in the validation cohort, we noted five misclassifications (17.2\%) (canonical correlation, 0.75; Wilks' $\lambda, 0.44$; $P<.001)$. The receiver operating characteristic area under the curve (95\% CI) for the entire group was 0.93 (0.86-0.99). When we added the variable age to the discriminatory analysis, the same three VOCs were chosen.

\section{Relationship Between VOCs and Clinical Characteristics}

Exhaled ammonia was associated with age $(\rho=0.41$, $P<.001)$, right atrial pressure $(\rho=0.57, P<.001)$, mean pulmonary artery pressure $(\rho=0.43, P=.015)$, cardiac index by thermodilution $(\rho=-0.39, P=.03)$, cardiac index by indirect Fick $(\rho=-0.38, P=.03)$, pulmonary vascular resistance $(\rho=0.40, P=.04), \mathrm{SvO}_{2}$ $(\rho=-0.59, P<.001)$, and right ventricular $(\mathrm{RV})$ dilation $(\rho=0.42, P=.03)$. Associations between ammonia and right atrial pressure or $\mathrm{SvO}_{2}$ remained significant when adjusted by age $(P<.001$ and $P=.002$, respectively) or age and $\operatorname{sex}(P<.001$ and $P=.02$, respectively). With the exception of pulmonary vascular resistance $(P=.11)$, all the previous associations between exhaled ammonia and hemodynamic measurements remained significant when we removed the two patients with liver disease. The receiver operating characteristic area under the curve for ammonia using patients with PAH vs healthy control subjects as the state variable was 0.74 (95\% CI, 0.61-0.87) when all patients with PAH were included and 0.78 (95\% CI, 0.65-0.9) when the two patients with portopulmonary hypertension were excluded.

The canonical score was associated with age $(\rho=-0.48, P<.001)$, mean pulmonary artery pressure $(\rho=0.4, P=.03), \mathrm{RV}$ function $(\rho=-0.44, P=.02)$, and RV dilation $(\rho=-0.44, P=.02)$. This variable remained a significant predictor of mean pulmonary artery pressure when adjusted by age $(P=.03)$.

Plasma ammonia was not different in patients with PAH $(\mathrm{n}=17)$ (median, $34 \mu \mathrm{mol} / \mathrm{L}$ [interquartile range, $21-49 \mu \mathrm{mol} / \mathrm{L}])$ and healthy control subjects $(\mathrm{n}=20)$ (median, $47 \mu \mathrm{mol} / \mathrm{L}$ [interquartile range, 31-53 $\mu \mathrm{mol} / \mathrm{L}$ ]) $(P=.3)$. There was no association between levels of ammonia in breath and plasma $(\rho=-0.01, P=.93)$. 
Table 2-Mass Scanning Peaks Corresponding to Ammonia

\begin{tabular}{|c|c|c|c|c|c|}
\hline Clinical Characteristic & $\mathrm{H}_{3} \mathrm{O}+18+$ & $\mathrm{NO}+17+$ & $\mathrm{NO}+18$ & $\mathrm{O}_{2}+17+$ & $\mathrm{O}_{2}+18+$ \\
\hline $\mathrm{RA}$ pressure, $\mathrm{mm} \mathrm{Hg}$ & $0.52,0.003$ & $\ldots$ & $\ldots$ & $0.38,0.04$ & $\ldots$ \\
\hline Mean PAP, $\mathrm{mm} \mathrm{Hg}$ & $0.43,0.02$ & $\ldots$ & $\ldots$ & $\ldots$ & $\ldots$ \\
\hline PVR, Wood Units & $0.42,0.02$ & $0.36,0.05$ & $\ldots$ & $\ldots$ & $\ldots$ \\
\hline Cardiac index, thermodilution $\mathrm{L} / \mathrm{min} / \mathrm{m}^{2}$ & $-0.66,0.001$ & $-0.5,0.005$ & $-0.46,0.01$ & $\ldots$ & $-0.38,0.04$ \\
\hline Cardiac index, indirect Fick L/min/m² & $-0.51,0.004$ & $\ldots$ & $-0.47,0.008$ & $\ldots$ & $\ldots$ \\
\hline $\mathrm{SvO}_{2}, \%$ & $-0.5,0.005$ & $-0.45,0.01$ & $-0.39,0.03$ & $-0.43,0.02$ & $-0.37,0.05$ \\
\hline
\end{tabular}

Data are presented as $\rho$ index, $P$ value. Only significant correlations are shown. PAP $=$ pulmonary artery pressure. See Table 1 legend for expansion of other abbreviations.

Similarly, there was no association between ammonia in plasma and clinical variables.

\section{Discussion}

This study demonstrates that patients with PAH have an exhaled breathprint that is different from that of healthy control subjects. Several specific VOCs were also significantly different between the groups (Table 3). Interestingly, volatile ammonia showed a significant direct association with hemodynamic and echocardiographic parameters, suggestive of more severe $\mathrm{PAH}$ disease.

Differences in the breath metabolic profile have been used previously to diagnose and classify diseases. ${ }^{15,23-25,27-35}$ Pattern recognition has been employed in sensor arrays for lung cancer screening ${ }^{28}$ and in the identification of patients with acute decompensated heart failure. ${ }^{36}$ In the current study, we used a patternrecognition approach on SIFT-MS to generate a specific breathprint that could distinguish accurately between healthy control subjects and patients with PAH.

Exhaled acetaldehyde and ethanol levels were higher in patients with PAH compared with control subjects (Table 3). The origin of acetaldehyde is probably the oxidation of ethanol produced by the gut microflora. It is interesting to speculate that the difference between acetaldehyde and ethanol in PAH compared with control breath is caused by the widespread metabolic dysfunction that has been noted in patients with PAH. The increase in these VOCs in the breath of patients with PAH may be caused by the altered nutrient metabolism associated with the gut microflora of patients with PAH. Indeed, diet-induced changes in the gut-flora-dependent metabolism can play an active of role in the pathogenesis of other diseases, including atherosclerosis. ${ }^{37}$ Another report indicated that acetaldehyde can be measured by SIFT-MS from the cell culture media of lung cancer cells. ${ }^{38}$ Although the precise reason for increased acetaldehyde production is not clear, acetaldehyde can be a by-product of the shift in glucose metabolism to aerobic glycolysis (the Warburg effect) seen in highly proliferative cells such as the vascular cells in PAH and cancer cells.

Exhaled breath levels of 2-propanol are typically associated with acetone, although at lower concentrations. Previous reports explain the combined detection of these compounds in exhaled breath in part by the reduction of acetone to 2-propanol. Our analysis of breath samples identified 2-propanol with no significant differences in levels of acetone between patients with PAH and healthy control subjects. In addition, 2-propanol levels were elevated in PAH breath samples. Recently, 2-propanol was found to be a discriminatory marker in exhaled breath condensate in cystic fibrosis and COPD. ${ }^{31,35}$ A more detailed biochemical assessment of this compound and its association with

Table 3-Volatile Organic Compounds in Patients With PAH and Healthy Control Subjects

\begin{tabular}{lccc}
\hline \hline Volatile Organic Compound & Patients With PAH, Median (IQR) & Healthy Control Subjects, Median (IQR) & $P$ Value, MWW \\
\hline No. & 31 & 34 & $\ldots$ \\
1-decene, ppb & $3.9(2-7)$ & $6(4-9)$ & .002 \\
1-octene, ppb & $10.3(4-17)$ & $15.8(11-23)$ & $.8(1-3)$ \\
2-nonene, ppb & $2.4(2-14)$ & $29.2(14-56)$ & .001 \\
2-propranol, ppb & $79.1(54-112)$ & $16.7(13-29)$ & $<.001$ \\
Acetaldehyde, ppb & $36.2(27-66)$ & $60.9(46-77)$ & $<.001$ \\
Ammonia, ${ }^{a}$ ppb & $94.7(70-129)$ & $49.1(25-68)$ & .001 \\
Ethanol, ppb & $75(53-130)$ & $11(8-14)$ & $<.001$ \\
Pentane, ppb & $16.8(12-23)$ & .001 \\
\hline
\end{tabular}

$\mathrm{IQR}=$ interquartile range; $\mathrm{MWW}=$ Mann-Whitney-Wilcoxon test; ppb = parts per billion. See Table 1 legend for expansion of other abbreviations. ahen we excluded the two patients with liver disease, the exhaled ammonia was 100 (76-131) ppb in patients with PAH and 61 (46-77) ppb in healthy control subjects $(P<.001)$. 
A

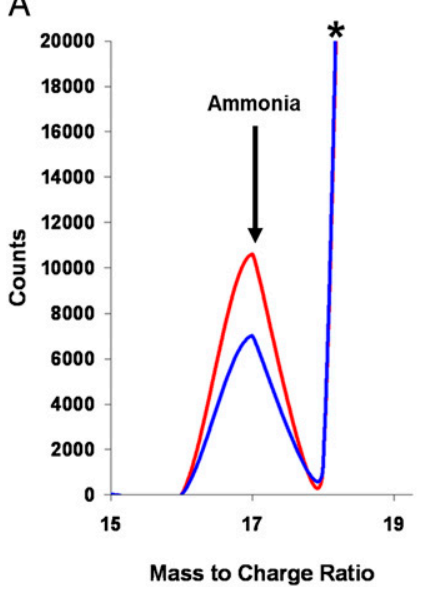

B

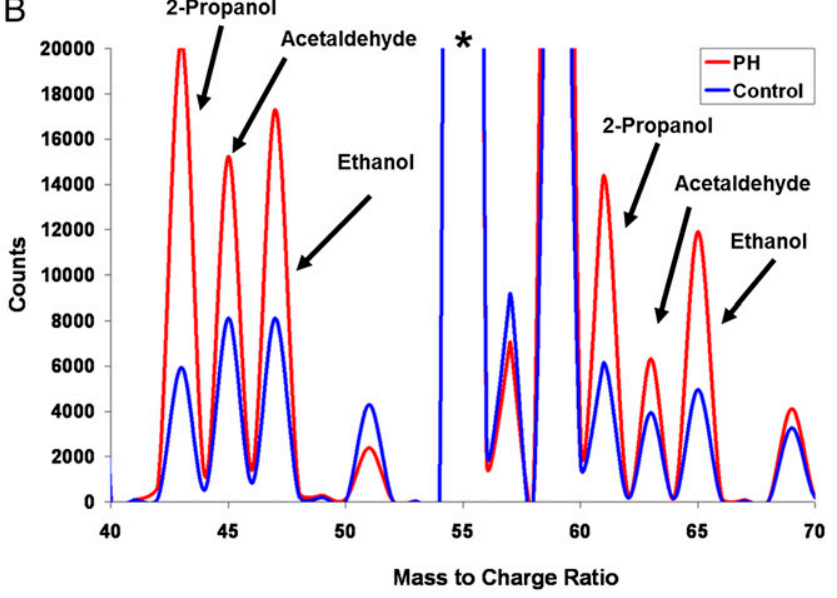

FiguRE 2. Representative areas of the average selected ion flow tube-mass spectrometry for the PAH and control groups. Labeled peaks correspond to selected compounds listed in Table 3. A, Ammonia peak comes from the $\mathrm{O}_{2}+$ precursor ion spectrum. B, Other molecules peaks from the $\mathrm{H}_{3} \mathrm{O}+$ precursor ion spectrum. The concentrations of a specific compound are calculated based on its counts relative to the precursor counts. *Precursor ion peak. PH = pulmonary hypertension. See Figure 1 legend for expansion of other abbreviation.

metabolic pathways within the disease need to be made.

Interestingly, plasma ammonia levels were no different from those of control subjects and had no significant correlation with the clinical parameters tested. However, increases in breath ammonia correlated positively with right atrial pressure, pulmonary artery pressure, and pulmonary vascular resistance. In addition, breath ammonia correlated negatively with cardiac index and $\mathrm{SvO}_{2}$. Increased breath ammonia may be a predictor of alterations in blood ammonia levels in PAH. Further work is needed to establish this connection.

Moreover, ammonia is generated from the breakdown of nucleic acids, polyamines, and amino acids, specifically glutamine. ${ }^{39}$ Highly proliferative cells undergo aerobic glycolysis and glutaminolysis instead of following the conventional oxidative phosphorylation pathway, resulting in a more rapid, albeit inefficient, use of glucose and glutamine for energy and nutrient synthesis. Because of this process, the rate of glutamine consumption can increase up to 10-fold in cancer cells compared with normal cells. ${ }^{40}$ Conspicuously, the lung has the highest glutamine levels of any tissue besides muscle. Metabolic changes that occur in the PAH lung may result in the use of these glutamine stores and increased glutaminolysis, much like that which happens in cancer cells. Thus, the metabolism of glutamine in highly proliferative PAH cells may generate the higher amounts of breath ammonia we have seen in this study. We believe that the association of breath ammonia with $\mathrm{PAH}$ disease severity suggests that this VOC may be an important PAH biomarker. Ultimately, increased breath ammonia levels may reflect not only the hemodynamic physiology, but also the underlying pathobiology of cellular proliferation.

Furthermore, studies have shown that use of glutamine in hexosamine biosynthesis can reduce nitric oxide synthesis through cellular depletion of nicotinamide adenine dinucleotide phosphate availability, an essential cofactor for nitric oxide synthase. ${ }^{39}$ This may explain, at least in part, the well-known nitric oxide deficiency state seen in PAH. ${ }^{41}$ Similarly, blood acid levels can be regulated by glutamine metabolism via the production of ammonia and bicarbonate. ${ }^{42}$ Regulation of blood $\mathrm{pH}$ levels through glutamine metabolism into ammonia may be a functional mechanism used by the body to govern the excess excretion of lactate, a product of both aerobic glycolysis and glutaminolysis. Further investigations of these phenomena are needed.

The limitations of our study include the relatively small sample size; PAH is a rare disease and we included only patients with PAH immediately after right-sided heart catheterization and prior to the initiation of therapy, to eliminate therapy as a confounding variable. Pulmonary hypertension is a heterogeneous disease with multiple causes. To minimize variability, we focused our studies only on patients with PAH. Although this does not eliminate the variability completely, it does minimize it significantly. Furthermore, the fact that we still found a consistent signal despite this variability offers reassurance that the signal is, in fact, there and that it likely reflects a common metabolic derangement in patients with PAH. In addition, the exhaled breath metabolome is complex and encompasses a large number of VOCs that need better characterization. 


\section{CONCLUSIONS}

Patients with PAH have an exhaled breathprint that is different from that of healthy control subjects. Higher levels of exhaled ammonia in patients with PAH correlate with severity of disease and likely reflect the metabolic changes associated with increased cellular proliferation in this disease.

\section{ACKNOWLEDGMENTS}

Author contributions: Dr Dweik is guarantor of the paper and takes responsibility for the integrity of the data and accuracy of the data analysis.

Mr Cikach: contributed to the design of the study, data collection, classification of patients, statistical analysis, interpretation of results, writing and critical revision of the manuscript for important intellectual content, and final approval of the manuscript submitted. Dr Tonelli: contributed to the design of the study, data collection, classification of patients, statistical analysis, interpretation of results, writing and critical revision of the manuscript for important intellectual content, and final approval of the manuscript submitted. Dr Barnes: contributed to the interpretation of results, writing and critical revision of the manuscript for important intellectual content, and final approval of the manuscript submitted.

Ms Paschke: contributed to the data collection, interpretation of results, and revision and final approval of the manuscript submitted. Ms Newman: contributed to the data collection, writing and critical revision of the manuscript for important intellectual content, and final approval of the manuscript submitted.

Dr Grove: contributed to the data collection, interpretation of results, writing and critical revision of the manuscript for important intellectual content, and final approval of the manuscript submitted. Dr Dababneh: contributed to the data collection, interpretation of results, writing and critical revision of the manuscript for important intellectual content, and final approval of the manuscript submitted.

Dr Wang: contributed to the interpretation of results and revision and final approval of the manuscript submitted.

Dr Dweik: contributed to the design of the study, classification of patients, interpretation of results, writing and critical revision of the manuscript, and final approval of the manuscript submitted.

Financial/nonfinancial disclosures: The authors have reported to CHEST that no potential conflicts of interest exist with any companies/organizations whose products or services may be discussed in this article.

Role of sponsors: The sponsors had no role in the design of the study, the collection and analysis of the data, or the preparation of the manuscript.

Additional information: The e-Figure can be found in the "Supplemental Materials" area of the online article.

\section{REFERENCES}

1. Badesch DB, Champion HC, Sanchez MA, et al. Diagnosis and assessment of pulmonary arterial hypertension. J Am Coll Cardiol. 2009;54(suppl 1):S55-S66.

2. McLaughlin VV, Archer SL, Badesch DB, et al; ACCF/AHA. ACCF/AHA 2009 expert consensus document on pulmonary hypertension: a report of the American College of Cardiology Foundation Task Force on Expert Consensus Documents and the American Heart Association: developed in collaboration with the American College of Chest Physicians, American Thoracic Society, Inc, and the Pulmonary Hypertension Association. Circulation. 2009;119(16):2250-2294.

3. Cracowski JL, Leuchte HH. The potential of biomarkers in pulmonary arterial hypertension. Am J Cardiol. 2012; 110(suppl 6):32S-38S.
4. Brown LM, Chen H, Halpern S, et al. Delay in recognition of pulmonary arterial hypertension: factors identified from the REVEAL Registry. Chest. 2011;140(1):19-26.

5. Rich S, Dantzker DR, Ayres SM, et al. Primary pulmonary hypertension. A national prospective study. Ann Intern Med. 1987;107(2):216-223.

6. Howard LS. Prognostic factors in pulmonary arterial hypertension: assessing the course of the disease. Eur Respir Rev. 2011;20(122):236-242.

7. McLaughlin VV, Archer SL, Badesch DB, et al; American College of Cardiology Foundation Task Force on Expert Consensus Documents; American Heart Association; American College of Chest Physicians; American Thoracic Society, Inc; Pulmonary Hypertension Association. ACCF/AHA 2009 expert consensus document on pulmonary hypertension a report of the American College of Cardiology Foundation Task Force on Expert Consensus Documents and the American Heart Association developed in collaboration with the American College of Chest Physicians; American Thoracic Society, Inc; and the Pulmonary Hypertension Association. J Am Coll Cardiol. 2009;53(17):1573-1619.

8. Benza RL, Gomberg-Maitland M, Miller DP, et al. The REVEAL Registry risk score calculator in patients newly diagnosed with pulmonary arterial hypertension. Chest. 2012; 141(2):354-362.

9. Pauling L, Robinson AB, Teranishi R, Cary P. Quantitative analysis of urine vapor and breath by gas-liquid partition chromatography. Proc Natl Acad Sci U S A. 1971;68(10): 2374-2376.

10. Dweik RA, Boggs PB, Erzurum SC, et al; American Thoracic Society Committee on Interpretation of Exhaled Nitric Oxide Levels (FENO) for Clinical Applications. An official ATS clinical practice guideline: interpretation of exhaled nitric oxide levels (FENO) for clinical applications. Am J Respir Crit Care Med. 2011;184(5):602-615.

11. Peng NJ, Lai KH, Lo GH, Hsu PI. Comparison of noninvasive diagnostic tests for Helicobacter pylori infection. Med Princ Pract. 2009;18(1):57-61.

12. Machado RF, Londhe Nerkar MV, Dweik RA, et al. Nitric oxide and pulmonary arterial pressures in pulmonary hypertension. Free Radic Biol Med. 2004;37(7):1010-1017.

13. Ozkan M, Dweik RA, Laskowski D, Arroliga AC, Erzurum SC. High levels of nitric oxide in individuals with pulmonary hypertension receiving epoprostenol therapy. Lung. 2001; 179(4):233-243.

14. Kaneko FT, Arroliga AC, Dweik RA, et al. Biochemical reaction products of nitric oxide as quantitative markers of primary pulmonary hypertension. Am J Respir Crit Care Med. 1998;158(3):917-923.

15. Paschke KM, Mashir A, Dweik RA. Clinical applications of breath testing. F1000 Med Rep. 2010;2:56.

16. Barnes PJ, Dweik RA, Gelb AF, et al. Exhaled nitric oxide in pulmonary diseases: a comprehensive review. Chest. 2010; 138(3):682-692.

17. Horvath I, Dweik R, Barnes PJ. Exhaled nitric oxide comes of age. J Breath Res; 2012;6(4):040201.

18. Grob NM, Dweik RA. Exhaled nitric oxide in asthma: progress since the introduction of standardized methodology. J Breath Res. 2008;2(3):37002.

19. Grob NM, Dweik RA. Exhaled nitric oxide in asthma. From diagnosis, to monitoring, to screening: are we there yet? Chest. 2008;133(4):837-839

20. Warwick G, Kotylar E, Chow S, et al. Exhaled breath condensate in pulmonary arterial hypertension. J Breath Res. 2012;6(3):036006.

21. Girgis REC, Champion HC, Diette GB, Johns RA, Permutt S, Sylvester JT. Decreased exhaled nitric oxide in pulmonary 
arterial hypertension: response to bosentan therapy. Am J Respir Crit Care Med. 2005;172(3):352-357.

22. Smith D, Spanel P. Selected ion flow tube mass spectrometry (SIFT-MS) for on-line trace gas analysis. Mass Spectrom Rev. 2005;24(5):661-700.

23. Braun PX, Gmachl CF, Dweik RA. Bridging the collaborative gap: realizing the clinical potential of breath analysis for disease diagnosis and monitoring- tutorial. Sensors Journal, IEEE. 2012;12(11):3258-3270.

24. Cikach FS Jr, Dweik RA. Cardiovascular biomarkers in exhaled breath. Prog Cardiovasc Dis. 2012;55(1):34-43.

25. Mashir A, Dweik RA. Exhaled breath analysis: the new interface between medicine and engineering. Adv Powder Technol. 2009;20(5):420-425.

26. Burns R, Burns R. Discriminatory Analysis. Business Research Methods and Statistics Using SPSS. Thousand Oaks, CA: Sage Publications Ltd; 2009:589-608.

27. Hunter GW, Dweik RA. Applied breath analysis: an overview of the challenges and opportunities in developing and testing sensor technology for human health monitoring in aerospace and clinical applications. J Breath Res. 2008;2(3):037020.

28. Machado RF, Laskowski D, Deffenderfer O, et al. Detection of lung cancer by sensor array analyses of exhaled breath. Am J Respir Crit Care Med. 2005;171(11):1286-1291.

29. Mazzone PJ, Hammel J, Dweik R, et al. Diagnosis of lung cancer by the analysis of exhaled breath with a colorimetric sensor array. Thorax. 2007;62(7):565-568.

30. Röck F, Barsan N, Weimar U. Electronic nose: current status and future trends. Chem Rev. 2008;108(2):705-725.

31. Montuschi P, Paris D, Melck D, et al. NMR spectroscopy metabolomic profiling of exhaled breath condensate in patients with stable and unstable cystic fibrosis. Thorax. 2012;67(3): 222-228.

32. Mashir A, Paschke KM, van Duin D, et al. Effect of the influenza A (H1N1) live attenuated intranasal vaccine on nitric oxide
$(\mathrm{FE}(\mathrm{NO}))$ and other volatiles in exhaled breath. J Breath Res. 2011;5(3):037107.

33. Hunter GW, Xu JC, Biaggi-Labiosa AM, et al. Smart sensor systems for human health breath monitoring applications. J Breath Res. 2011;5(3):037111.

34. Dweik RA. The great challenge for exhaled breath analysis: embracing complexity, delivering simplicity. J Breath Res. 2011;5(3):030201.

35. de Laurentiis G, Paris D, Melck D, et al. Separating smokingrelated diseases using NMR-based metabolomics of exhaled breath condensate. J Proteome Res. 2013;12(3):1502-1511.

36. Samara MA, Tang WHW, Cikach FS Jr, et al. Single exhaled breath metabolomic analysis identifies unique breathprint in patients with acute decompensated heart failure. J Am Coll Cardiol. 2013;61(13):1463-1464.

37. Tang WH, Wang Z, Levison BS, et al. Intestinal microbial metabolism of phosphatidylcholine and cardiovascular risk. N Engl J Med. 2013;368(17):1575-1584.

38. Rutter AV, Chippendale TWE, Yang Y, Španěl P, Smith D, Sulé-Suso J. Quantification by SIFT-MS of acetaldehyde released by lung cells in a 3D model. Analyst (Lond). 2013; 138(1):91-95.

39. Wu G, Haynes TE, Li H, Yan W, Meininger CJ. Glutamine metabolism to glucosamine is necessary for glutamine inhibition of endothelial nitric oxide synthesis. Biochem J. 2001; 353 (pt 2):245-252.

40. Deberardinis RJ, Sayed N, Ditsworth D, Thompson CB. Brick by brick: metabolism and tumor cell growth. Curr Opin Genet Dev. 2008;18(1):54-61.

41. Tonelli AR, Haserodt S, Aytekin M, Dweik RA. Nitric oxide deficiency in pulmonary hypertension: pathobiology and implications for therapy. Pulm Circ. 2013;3(1):20-30.

42. Taylor L, Curthoys NP. Glutamine metabolism: role in acid-base balance*. Biochem Mol Biol Educ. 2004;32(5): 291-304. 